\title{
La diabetes tipo 2 puede prevenirse con dieta y ejercicio
}

Prevention of type 2 diabetes mellitus by changes in lifestyle among subjects with impaired glucose tolerance. Tuomilehto $\mathrm{J}$, Linsdtrom $\mathrm{J}$, Eriksson J, et al. (Finnish Diabetes Prevention Study). N Engl J Med 2001;344:1343-50.

\section{Objetivo}

Estudiar el efecto de cambios en el estilo de vida de pacientes con alto riesgo de diabetes sobre la incidencia de esta enfermedad.

\section{Diseño}

Ensayo clínico controlado aleatorizado.

\section{Lugar}

Cinco Centros de Salud en Helsinki, Finlandia.

\section{Pacientes}

Se incluyeron 523 sujetos con un índice de masa corporal (IMC) mayor o igual a 25, de entre 40 a 65 años, y presencia de intolerancia oral a la glucosa (IOG). La presencia de diabetes fue un criterio de exclusión. Se definió IOG como la presencia de una glucemia de entre 140 a $200 \mathrm{mg} / \mathrm{dl}$ luego de 2 horas de ingesta de $75 \mathrm{gr}$. de glucosa medido en dos oportunidades, en sujetos con glucemias normales en ayunas. La media de seguimiento fue de 3,2 años.

\section{Intervención}

Los sujetos fueron aleatorizados al grupo intervención o control. Los sujetos del grupo control recibían información general oral y escrita sobre dieta y ejercicio inicialmente y en visitas una vez por año, donde también se evaluaba la alimentación mediante una cartilla de registro. En el grupo intervención, se daba información detallada sobre dieta, y el cumplimiento de cinco metas: descenso de peso de por lo menos el $5 \%$, ingesta de grasa menor al $30 \%$ de la energía consumida, ingesta de grasas saturadas menor al $10 \%$ de la energía consumida, ingesta de fibras mayor o igual a 15 gramos cada 1000 cal. y actividad física como mínimo liviana (caminar o andar en bicicleta) por lo menos 4 horas por semana.
Cada sujeto tenía siete entrevistas programadas con una nutricionista durante el primer año, y el cumplimiento de la dieta estaba supervisado e individualizado. Se les ofrecía programas de actividad física individualizados.

\section{Medición de resultados principales}

El resultado principal fue el diagnóstico de diabetes medido una vez por año, como un nivel de glucemia en ayunas mayor o igual a $140 \mathrm{mg} / \mathrm{dl}$, o una prueba de tolerancia oral a la glucosa de 200 $\mathrm{mg} / \mathrm{dl}$ o mayor luego de 2 horas de una ingesta de sobrecarga oral de glucosa. El diagnóstico requería ser confirmado por una segunda prueba. Se evaluó el grado de cumplimiento de las cinco metas.

\section{Resultados Principales}

La reducción de peso al segundo año (media+/-DS) fue de $3.5+/-5.5 \mathrm{~kg}$ en el grupo intervención y $0,8+/-4.4$ en el grupo control $(p<0,001)$. La incidencia acumulativa de diabetes luego de cuatro años fue de $10 \%$ en el grupo intervención y $23 \%$ en el grupo control. El riesgo de diabetes se redujo un $56 \%$ (IC 95\% 33 a 71 , $\mathrm{p}<0.001$ ) en el grupo intervención. El NNT* es de 8 (IC 95\% 5 a 15).

El grado de cumplimiento de las 5 metas fue significativamente superior para el grupo intervención que para el control. Se encontró una correlación* negativa entre el número de metas cumplidas y la incidencia de diabetes. Amayor número de metas cumplidas, menor incidencia de diabetes. En los sujetos que cumplieron 4-5 metas no se encontró ningún caso de diabetes.

\section{Conclusiones}

En sujetos de alto riesgo, la diabetes tipo 2 puede prevenirse mediante cambios en el estilo de vida.

\section{Comentario}

Las personas con antecedentes familiares de diabetes tipo 2 , diabetes gestacional, obesidad o glucemia en ayunas alterada, tienen un mayor riesgo de desarrollar diabetes mellitus.

La diabetes mellitus ha triplicado su prevalencia en los últimos 30 años con enormes consecuencias en la salud pública. De manera que la posibilidad de realizar prevención primaria de la enfermedad constituye un enorme desafío.

Este ensayo de Tuomiletho, aporta evidencia acerca de la eficacia de cambios en el estilo de vida para prevenir que pacientes de alto riesgo desarrollaran diabetes. Sus hallazgos son coincidentes con el estudio realizado en Da Qing (China) en el cual ya se había demostrado que el riesgo de desarrollar diabetes podía reducirse 1. Un ensayo clínico aún no publicado realizado en EE.UU (Diabetes Prevention Study) ${ }^{2}$, pero cuyos resultados están disponibles, ha demostrado que un programa de dieta para reducir un $7 \%$ del peso y 150 minutos de ejercicios por semana también redujo la incidencia de diabetes en un $58 \%$. Lo interesante de este último trabajo es que se llevó a cabo en 3.234 pacientes y que incluyó minorías étnicas (negros, hispanos) y mayores de 60 años con lo que sus conclusiones son más generalizables.

Si bien toda la evidencia es concordante y concluyente (la diabetes puede prevenirse), los médicos de atención primaria sabemos cuán difícil es lograr que las personas adhieran a cambios del estilo de vida. Sin embargo, si conocemos sus beneficios en términos de morbilidad -podríamos prevenir un caso de diabetes por cada ocho pacientes que intervenimos a lo largo de tres años-, es probable que aumentemos nuestros esfuerzos en educar, concientizar y lograr adherir a los pacientes. 